\title{
Queuing Analysis of Dynamic Spectrum Access Subject to Interruptions from Primary Users
}

\author{
Husheng Li and Zhu Han
}

\begin{abstract}
Interruption from primary users is a key factor impacting the performance of secondary users in cognitive radio networks. Based on a simplified model of primary user interruptions (Markov chain), queuing analysis is carried out for two-server-single-queue and single-server-two-queue cases. A semi-analytic result is obtained for the generating function of queue length in the two-server-single-queue case. The average queue length is obtained from solving a group of linear equations for the single-server-two-queue case. The maximal packet arrival rates versus different service rates and interruption parameters are obtained via numerical simulations.
\end{abstract}

\section{INTRODUCTION}

In recent years, cognitive radio has received intensive studies due to its capability of efficiently utilizing frequency spectrum [6]. In a cognitive radio system, a secondary user (without license to the spectrum band) can access the spectrum if it detects no existence of primary users (with license of the band). Therefore, the spectrum resource can be efficiently used, particular in areas where primary users are scarce.

Currently, research on cognitive radio is mainly focused on spectrum sensing, i.e. detecting the existence of primary users [7], channel selection, i.e. selecting the optimal channel(s) for sensing due to limited capability of sampling [13] and resource allocation, i.e. how to allocate the available spectrum resources among multiple secondary users [5].

However, the above researches typically assume full buffers, i.e. there always exists data for transmission, and ignore the burstiness of data traffics, which requires queuing analysis if the spectrum is considered as a server and the data packets in secondary user are regarded as customers. Such a queuing analysis in cognitive radio systems is still lacking although some pioneering literatures have been published recently [1] [8] [9] [11].

In this paper, we study the queuing analysis for secondary users dynamically accessing spectrum in cognitive radio systems, particularly for special cases of single-queue-two-server and two-queue-single-server, respectively. The feature that distinguishes cognitive radio from traditional wireless networks is that the server(s) is interrupted by primary users since the service for secondary users has to be stopped once primary users emerge. Queuing analysis with interrupted server(s) (also known as vacation model) is originated by D. P. Gaver in

H. Li is with the Department of Electrical Engineering and Computer Science, the University of Tennessee, Knoxville, TN, 37996 (email husheng@eecs.utk.edu). Z. Han is with the Department of Electrical and Computer Engineering, University of Houston, Houston, TX, 77004. This work was supported by the National Science Foundation under grants $\mathrm{CCF}$ 0830451, CNS-0905556, CNS-0953377, CNS-0910461 and ECCS-0901425.
1962 [4] for single queue and single server case. The analysis is extended to more complicated situations in subsequent years [2] [3] [12]. Note that the interrupted sever model is also used in [1] for the queuing analysis in cognitive radio systems. However, different from [1], we do not explicitly model primary users and we consider the possible confliction between different secondary users, which is more reasonable in practical systems, in this paper.

Actually, interruption becomes a new factor impacting the system performance in cognitive radio systems, besides the traditional ones, namely fading and interference. The differences between interruption and interference/fading are summarized below:

- Interruption and fast fading: interruption can be somewhat considered as deep fading since no information can be transmitted during the interruption period. However, different from fading, interruption is more temporally and spatially correlated. There is more predicability (i.e. using the statistical feature of data burst like self-similarity) for interruption than the random fading. Moreover, fast fading can beneficial (when signal is strengthened), while interruption is always harmful.

- Interruption and interference: essentially, interruption arises from interference. However, interruption does not need strong interference since the secondary user needs to quit the channel even when the detected signal of primary user is very weak (e.g. the detection threshold for DTV is $-116 \mathrm{dBm}$ in IEEE 802.22 standard, which is comparable to noise power). Moreover, interference across different users can be alleviated by interference avoidance or multiple access schemes while interruption cannot due to the absolute priority of primary users.

The remainder of this paper is organized as follows. The system model is introduced in Section II. The queuing analysis is carried out for two-server case and two-queue case in Sections III and IV, respectively. Numerical results and conclusions are given in Sections V and VI, respectively.

\section{System Model}

We consider licensed frequency bands and the emergence of primary users as servers and interruption, respectively. Then, the data packets are equivalent to customers in queue(s). The discrete timing structure of the queuing system is illustrated in Fig. 1, where packets arrive at the beginning of each spectrum access period and secondary user(s) receives service subsequently. The buffer length is counted at the end of each spectrum access period. 


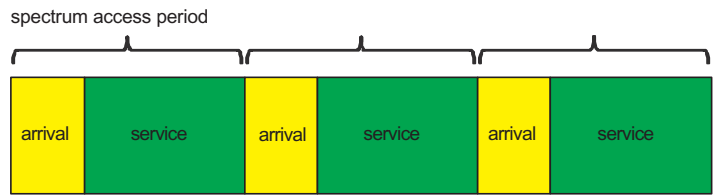

$\stackrel{\text { time }}{\longrightarrow}$

Fig. 1: Timing structure of each spectrum access period.

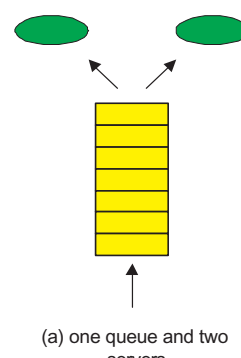
servers

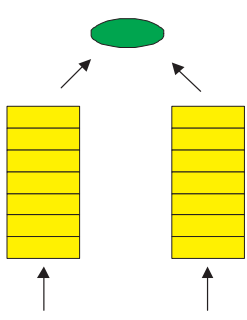

(b) two queues and one server
Fig. 2: Illustration of the situations studied in this paper.

We consider two scenarios, namely single queue (a single secondary user) and two servers (two licensed channels), shown in Fig. 2 (a), and two queues (two secondary users) and single server (single channel), shown in Fig. 2 (b). The details of spectrum access will be introduced in Sections III and IV, respectively.

The following assumptions are placed throughout the paper, unless otherwise stated.

- The arrival of packet is Bernoulli, i.e. there is at most one packet arriving in each spectrum access period and the packet arrival probability is denoted by $\lambda$.

- The service is also Bernoulli, i.e. at most one packet can finish its service in each spectrum access period and the corresponding success probability is denoted by $\mu$.

- The emergence of primary user (interruption) is Markovian, i.e. each server has two states, busy (B, the channel is occupied by primary users) or idle ( $\mathrm{I}$, there is no primary user), and the transition between the two states is Markovian. We define the a posteriori probability of previous state conditioned on current state as

$$
\begin{aligned}
Q_{s_{1} s_{2}}(t) & =P\left(S_{t}=s_{1} \mid S_{t+1}=s_{2}\right) \\
& =\frac{P\left(S_{t+1}=s_{2} \mid S_{t}=s_{1}\right) P\left(S_{t}=s_{1}\right)}{P\left(S_{t+1}=s_{2}\right)},(1)
\end{aligned}
$$

where $S_{t}$ means the state at period $t$.

Note that the first two assumptions are for the simplicity of analysis and can be extended to general arrival process. It is difficult to relax the assumption of Markovian interruptions to more general case (e.g. semi-Markov process) since the computational complexity will be substantially increased.

\section{AnAlysis of Two-Server-Single-Queue CASE}

In this section, we discuss two-server-single-queue case using semi-analytic approach. We assume that the interruption processes, as well as the service processes, are mutually independent at these two servers. For simplicity, we assume that the two servers are symmetric, i.e. the parameters of the corresponding interruptions and services are the same for the two servers.

We define conditional generating function $G_{s_{1} s_{2}}(z)$ of the queue length, conditioned that the two channels are in states $s_{1}$ and $s_{2}$, which is given by

$$
G_{s_{1} s_{2}}(z)=\sum_{l=0}^{\infty} P\left(X_{t}=l \mid S_{t}^{1}=s_{1}, S_{t}^{2}=s_{2}\right) z^{l},
$$

where $X_{t}$ is the queue length at an arbitrary spectrum access period $t$ and $S_{t}^{i}$ denotes the state of server $i, i=1,2$. We denote by $A(z)$ the generating function of arrival packets, which is given by $A(z)=(1-\lambda)+\lambda z$, according to the assumption of Bernoulli arrivals. We also define the operator for the probability of successfully serving one packet, which is given by

$$
L(z)=\mu z^{-1}+(1-\mu) .
$$

When the queuing process is stable, it is easy to check

$$
\begin{aligned}
G_{B B}(z) & =\left(Q_{B B}^{2} G_{B B}(z)+Q_{B B} Q_{I B} G_{B I(z)}\right. \\
& \left.+Q_{B B} Q_{I B} G_{I B}(z)+Q_{I B}^{2} G_{I I}(z)\right) A(z),
\end{aligned}
$$

and

$$
\begin{aligned}
G_{B I}(z) & =\left(Q_{B B} Q_{B I} G_{B B}(z)+Q_{B B} Q_{I I} G_{B I(z)}\right. \\
& \left.+Q_{I B} Q_{B I} G_{I B}(z)+Q_{I B} Q_{I I} G_{I I}(z)\right) \\
& \times L(z) A(z)+D_{B I}(1-L(z)),
\end{aligned}
$$

where $D_{B I}$ is a constant equaling the probability of empty queue when the server states are busy and idle, respectively, which is given by

$$
\begin{aligned}
D_{B I} & =A(z)\left(Q_{B B} Q_{B I} G_{B B}(z)+Q_{B B} Q_{I I} G_{B I}(z)\right. \\
& \left.+Q_{I B} Q_{B I} G_{I B}(z)+Q_{I B} Q_{I I} G_{I I}(z)\right)\left.\right|_{z=0},
\end{aligned}
$$

and

$$
\begin{aligned}
G_{I B}(z) & =\left(Q_{B B} Q_{B I} G_{B B}(z)+Q_{B I} Q_{I B} G_{B I(z)}\right. \\
& \left.+Q_{I I} Q_{B B} G_{I B}(z)+Q_{I B} Q_{I I} G_{I I}(z)\right) \\
& \times L(z) A(z)+D_{I B}(1-L(z)),
\end{aligned}
$$

where $D_{I B}=D_{B I}$, and

$$
\begin{aligned}
G_{I I}(z) & =\left(Q_{B I}^{2} G_{B B}(z)+Q_{B I} Q_{I I} G_{B I}(z)\right. \\
& \left.+Q_{I I} Q_{B I} G_{I B}(z)+Q_{I I}^{2} G_{I I}(z)\right) L^{2}(z) A(z) \\
& +D_{I I}\left(1-L^{2}(z)\right)+E_{I I} z\left(L(z)-L^{2}(z)\right),
\end{aligned}
$$

where $D_{I I}$ is a constant equaling the probability of empty queue when the server states are both idle, which is given by

$$
\begin{aligned}
D_{I I}(z) & =A(z)\left(Q_{B I}^{2} G_{B B}(z)+Q_{B I} Q_{I I} G_{B I(z)}\right. \\
& \left.+Q_{I I} Q_{B I} G_{I B}(z)+Q_{I I}^{2} G_{I I}(z)\right)\left.\right|_{z=0} .
\end{aligned}
$$


and $E_{I I}$ is equal to the probability that there is only one packet in the queue right after the arrival stage when the server states are both idle

$$
\begin{aligned}
E_{I I} & =\frac{d}{d z} A(z)\left(Q_{B I}^{2} G_{B B}(z)+Q_{B I} Q_{I I} G_{B I(z)}\right. \\
& \left.+Q_{I I} Q_{B I} G_{I B}(z)+Q_{I I}^{2} G_{I I}(z)\right)\left.\right|_{z=0} .
\end{aligned}
$$

The above equations can be written as

$$
\mathbf{g}(z)=\mathbf{B}(z) \mathbf{g}(z)+\mathbf{C}(z) \mathbf{f},
$$

where

$$
\mathbf{g}(z)=\left(G_{B B}(z), G_{B I}(z), G_{I B}(z), G_{I I}(z)\right)^{T},
$$

$\mathbf{B}(z)$ is defined in (13) (next page) and

$\mathbf{C}(z)=\left(\begin{array}{cccc}0 & 0 & 0 & 0 \\ 0 & 1-L(z) & 0 & 0 \\ 0 & 1-L(z) & 0 & 0 \\ 0 & 0 & 1-L^{2}(z) & z\left(L(z)-L^{2}(z)\right)\end{array}\right)$,

and $\mathbf{f}$ is a constant, which is given by

$$
\mathbf{f}=\left(0, D_{I B}, D_{I I}, E_{I I}\right)^{T} .
$$

Then, we obtain the expression of the generating function vector $\mathrm{g}$

$$
\mathbf{g}=(\mathbf{I}-\mathbf{B}(z))^{-1} \mathbf{C}(z) \mathbf{f} .
$$

Then then unconditional generating function of queue length (independent of server states) can be obtained from the weighted sum of these conditional generating functions (the weights are the probabilities of the server states). However, we are still unable to obtain explicit expressions for the constants in $\mathbf{f}$. Currently, we use simulations to obtain $\mathbf{f}$.

\section{Analysis of Two-Queue-Single-Server CASE}

In this section, we analyze the case of two queues and a single server. We assume that the server can server only one queue at a time and collision is solved similarly to the combination of $p$-persistent CSMA and Aloha, namely, when a secondary user has new data packet, it transmit with probability $p$. If both secondary users transmit simultaneously, a collision happens and no packet gets through. Note that, the multiple access scheme used here is different from standard asynchronous CSMA, the analysis can be easily extended to the case of asynchronous CSMA using embedded Markov chain similarly to [10].

\section{A. Generating Function}

Similarly to the previous section, we define the generating function of packet arrival as

$$
A\left(z_{1}, z_{2}\right)=\left(\lambda z_{1}+1-\lambda\right)\left(\lambda z_{2}+1-\lambda\right),
$$

and the operator of service for one queue

$$
L_{1}(z)=\mu p z^{-1}+1-\mu p,
$$

and the operator of service for two queues

$$
\begin{aligned}
L_{2}\left(z_{1}, z_{2}\right) & =\mu p(1-p) z_{1}^{-1}+\mu p(1-p) z_{2}^{-1} \\
& +1-2 \mu p(1-p) .
\end{aligned}
$$

We also define the conditional generating functions of queue lengths $G_{s}\left(z_{1}, z_{2}\right)$, where $s$ is the server state, similarly to (2). Then, it is easy to verify that, when the queuing process becomes stable, the conditional generating functions are given by

$G_{B}\left(z_{1}, z_{2}\right)=\left(Q_{B B} G_{B}\left(z_{1}, z_{2}\right)+Q_{I B} G_{I}\left(z_{1}, z_{2}\right)\right) A\left(z_{1}, z_{2}\right)$,

and

$$
\begin{aligned}
& G_{I}\left(z_{1}, z_{2}\right) \\
= & Q_{B I}\left\{G_{B}(0,0) A(0,0)\right. \\
+ & {\left[G_{B}\left(z_{1}, 0\right) A\left(z_{1}, 0\right)-G_{B}(0,0) A(0,0)\right] L_{1}\left(z_{1}\right) } \\
+ & {\left[G_{B}\left(0, z_{2}\right) A\left(0, z_{2}\right)-G_{B}(0,0) A(0,0)\right] L_{1}\left(z_{2}\right) } \\
+ & \left(G_{B}\left(z_{1}, z_{2}\right) A\left(z_{1}, z_{2}\right)-G_{B}\left(z_{1}, 0\right) A\left(z_{1}, 0\right)\right. \\
- & \left.\left.G_{B}\left(0, z_{2}\right) A\left(0, z_{2}\right)+G_{B}(0,0) A(0,0)\right) L_{2}\left(z_{1}, z_{2}\right)\right\} \\
+ & Q_{I I}\left\{G_{I}(0,0) A(0,0)\right. \\
+ & {\left[G_{I}\left(z_{1}, 0\right) A\left(z_{1}, 0\right)-G_{I}(0,0) A(0,0)\right] L_{1}\left(z_{1}\right) } \\
+ & {\left[G_{I}\left(0, z_{2}\right) A\left(0, z_{2}\right)-G_{I}(0,0) A(0,0)\right] L_{1}\left(z_{2}\right) } \\
+ & \left(G_{I}\left(z_{1}, z_{2}\right) A\left(z_{1}, z_{2}\right)-G_{I}\left(z_{1}, 0\right) A\left(z_{1}, 0\right)\right. \\
- & \left.\left.G_{I}\left(0, z_{2}\right) A\left(0, z_{2}\right)+G_{B}(0,0) A(0,0)\right) L_{2}\left(z_{1}, z_{2}\right)\right\} \cdot(21)
\end{aligned}
$$

Defining $\mathbf{g}=\left(G_{B}\left(z_{1}, z_{2}\right), G_{I}\left(z_{1}, z_{2}\right)\right)^{T}$, we can rewrite (20) and (21) in the following vector form:

$$
\mathbf{g}=A \mathbf{B g}+\mathbf{h}+\mathbf{o}
$$

where

$$
\mathbf{B}=\left(\begin{array}{cc}
Q_{B B} & Q_{I B} \\
Q_{B I} L_{2}\left(z_{1}, z_{2}\right) & Q_{I I} L_{2}\left(z_{1}, z_{2}\right)
\end{array}\right),
$$

and

$$
\begin{aligned}
\mathbf{h} & =\left(0, Q_{B I} G_{B}\left(z_{1}, 0\right) A\left(z_{1}, 0\right)\left(L_{1}\left(z_{1}\right)-L_{2}\left(z_{1}, z_{2}\right)\right)\right. \\
& +Q_{B I} G_{B}\left(0, z_{2}\right) A\left(0, z_{2}\right)\left(L_{1}\left(z_{2}\right)-L_{2}\left(z_{1}, z_{2}\right)\right) \\
& +Q_{I I} G_{I}\left(z_{1}, 0\right) A\left(z_{1}, 0\right) L_{1}\left(\left(z_{1}\right)-L_{2}\left(z_{1}, z_{2}\right)\right) \\
& \left.+Q_{I I} G_{I}\left(0, z_{2}\right) A\left(0, z_{2}\right)\left(L_{1}\left(z_{2}\right)-L_{2}\left(z_{1}, z_{2}\right)\right)\right) \cdot(24)
\end{aligned}
$$

and

$$
\begin{aligned}
\mathbf{o} & =\left(0, Q_{B I} G_{B}(0,0) A(0,0) L^{\prime}\left(z_{1}, z_{2}\right)\right. \\
& \left.+Q_{I I} G_{I}(0,0) A(0,0) L^{\prime}\left(z_{1}, z_{2}\right)\right),
\end{aligned}
$$

where $L^{\prime}\left(z_{1}, z_{2}\right)=1-L_{1}\left(z_{1}\right)-L_{1}\left(z_{2}\right)+L_{2}\left(z_{1}, z_{2}\right)$.

Then, we have

$$
\mathbf{g}=\left(\mathbf{I}-A\left(z_{1}, z_{2}\right) \mathbf{B}\right)^{-1}(\mathbf{h}+\mathbf{o}) .
$$




$$
\mathbf{B}(z)=A(z)\left(\begin{array}{cccc}
Q_{B B}^{2} & Q_{B B} Q_{I B} & Q_{B B} Q_{I B} & Q_{I B}^{2} \\
Q_{B B} Q_{B I} L(z) & Q_{B B} Q_{I I} L(z) & Q_{I B} Q_{B I} L(z) & Q_{I B} Q_{I I} L(z) \\
Q_{B B} Q_{B I} L(z) & Q_{B I} Q_{I B} L(z) & Q_{I I} Q_{B B} L(z) & Q_{I I} Q_{I B} L(z) \\
Q_{B I}^{2} L^{2}(z) & Q_{B I} Q_{I I} L^{2}(z) & Q_{I I} Q_{B I} L^{2}(z) & Q_{I I}^{2} L^{2}(z)
\end{array}\right)
$$

\section{B. Average Queue Length}

Although we obtained an equation characterizing the generating function of queue length, it is prohibitively to obtain the explicit expression of $\mathbf{g}$ since the right hand side of (26) is also unknown. However, we manage to obtain the average queue length, which equals $\mathbf{g}_{1}(1,0)$, where $\mathbf{g}_{1}\left(z_{1}, z_{2}\right) \triangleq \frac{\partial \mathbf{g}}{\partial z_{1}}\left(z_{1}, z_{2}\right)$. In the sequel, we derive six equations from which we can obtain six unknowns in $\mathbf{g}_{1}(1,0), \mathbf{g}(1,0)$ and $\mathbf{g}(0,0)$.

1) Equations 1 and 2: We obtain the following equation by taking partial derivative with respect to $z_{1}$ on both sides of (22):

$$
\begin{aligned}
& (\mathbf{I}-(1-\lambda) \mathbf{B}) \mathbf{g}_{1}(1,0) \\
-\quad & \lambda(1-\lambda)(\mathbf{B}-\mu \mathbf{C}) \mathbf{g}(1,0) \\
+\quad & (1-\lambda)^{2}\left(2 \mu p-\mu p^{2}\right) \mathbf{C g}(0,0)=0,
\end{aligned}
$$

where

$$
\mathbf{C}=\left(\begin{array}{cc}
0 & 0 \\
Q_{B I} & Q_{I I}
\end{array}\right)
$$

2) Equations 3 and 4: It is easy to verify that

$$
\left.\frac{d \mathbf{g}(z, z)}{d z}\right|_{z=1}=2 \mathbf{g}_{1}(1,0) \text {. }
$$

Then, letting $z_{1}=z_{2}=z$ and taking derivative with respect to $z$ on both sides of (22), we have

$$
\begin{aligned}
& (\mathbf{I}-\mathbf{B}) \mathbf{g}_{1}(1,0) \\
-\quad & \lambda(1-\lambda) \mathbf{C g}(1,0) \\
+\quad & (1-\lambda)^{2}\left(2 \mu p-\mu p^{2}\right) \mathbf{C g}(0,0)=\lambda \mathbf{1} .
\end{aligned}
$$

where we applied the facts $\mathbf{g}(1,1)=\mathbf{1}, G_{B}(1,0)=$ $G_{B}(0,1), G_{I}(1,0)=G_{I}(0,1)$ and $\mathbf{B 1}=\mathbf{1}$.

3) Equation 5: Substituting $z_{1}=z_{2}=0$ into (20), we have

$G_{B}(0,0)=\left(Q_{B B} G_{B}(0,0)+Q_{I B} G_{I}(0,0)\right)(1-\lambda)^{2}$.

4) Equation 6: Substituting $z_{1}=z_{2}=1$ into (26) and applying the fact $G_{I}(1,1)=1$, we have

$$
\mathbf{1}=\lim _{z_{1} \rightarrow 1}\left(\mathbf{I}-A\left(z_{1}, 1\right) \mathbf{B}\right)^{-1}\left(\mathbf{h}\left(z_{1}, 1\right)+\mathbf{o}\left(z_{1}, 1\right)\right) .
$$

Then, we have

$$
\begin{gathered}
(1-\lambda)\left(C_{1}+C_{2}\right)\left(Q_{B I} G_{B}(1,0)+Q_{I I} G_{I}(1,0)\right) \\
-\quad(1-\lambda)^{2} C_{1}\left(Q_{B I} G_{B}(0,0)+Q_{I I} G_{I}(0,0)\right)=1,(33)
\end{gathered}
$$

where

$$
\begin{aligned}
C_{1} & =\lim _{z_{1} \rightarrow 1} \frac{\left(1-A\left(z_{1}, 1\right) Q_{B B}\right)\left(L_{1}\left(z_{1}\right)-L_{2}\left(z_{1}, 1\right)\right)}{\operatorname{det}(\mathbf{I}-A \mathbf{B})} \\
& =-\frac{\left(1-Q_{B B}\right) u p^{2}}{\left.\frac{d \operatorname{det}(\mathbf{I}-A \mathbf{B})}{d z_{1}}\right|_{z_{1}=1}} \\
& =\frac{\left(1-Q_{B B}\right) u p^{2}}{\lambda\left(Q_{B I}+Q_{I B}\right)-u p(1-p)\left(Q_{I B} Q_{I I}+Q_{I B} Q_{B I}\right)}
\end{aligned}
$$

$$
\begin{aligned}
& \text { and } \\
& \begin{aligned}
C_{2} & =\lim _{z_{1} \rightarrow 1} \frac{\left(1-A\left(z_{1}, 1\right) Q_{B B}\right)\left(a(1)-b\left(z_{1}, 1\right)\right)}{\operatorname{det}(\mathbf{I}-A \mathbf{B})} \\
& =-\frac{\left(1-Q_{B B}\right) u p(1-p)}{\lambda\left(Q_{B I}+Q_{I B}\right)-u p(1-p)\left(Q_{I B} Q_{I I}+Q_{I B} Q_{B I}\right)}
\end{aligned}
\end{aligned}
$$

Combining the above six equations (27)(30)(31)(33), we can obtain $\mathbf{g}_{1}(1,0)$, from which we obtain the average queue length. As byproducts, we obtain $\mathbf{g}(0,0)$, which is the probability that both queues are empty, and $\mathbf{g}(1,0)$, which is the probability that at least one queue is empty.

\section{NUMERICAL RESUlTS}

In this section, we use numerical simulations to test the stability of the queuing system under interruptions of primary users. We focused on the stability of the queuing system, i.e. the maximum arrival rate supported by the servers, since the queue length in stable situations can be analyzed using the analytic (or semi-analytic) conclusions developed in Sections III and (IV).

\section{A. Two-server-single-queue Case}

Figures 3 and 4 show the maximal arrival rate $\lambda$ supported by the queuing system versus different service rates $\mu$ and different interruption parameters. In Fig. 3, we vary the probability of transiting from busy state to idle state, denoted by $P_{B I}$. The higher $P_{B I}$ is, the shorter interruption duration is (in the statistical sense). We observe that larger $P_{B I}$ incurs lower supportable packet arrival rate; however, the performance degradation is not dramatic. In Fig. 4, we vary the probability of transmitting from idle state to idle state, denoted by $P_{I I}$. The higher $P_{I I}$ is, the less interruptions arrive. Again, we observe that more interruptions reduce the supportable packet arrival rate and the performance degrades rapidly.

\section{B. Two-queue-single-server Case}

In Fig. 5, we show the maximal packet arrival rate that can be supported by a two-queue-single-server queuing system, based on the optimal transmission probability $p$ (obtained from exhaustive search). We observe that, even when $\mu=1$ and $P_{I I}=1$ (no interruption), the maximal packet arrival rate is significantly less than 1 due to the collision between the two queues. Fig. 6 shows the optimal transmit probability corresponding to the maximal packet arrival rate shown in Fig. 5. We observe that, when there is no interruption $\left(Q_{I I}=1\right)$, the optimal transmit probability is an increasing function of $\mu$. However, when interruption exists, the monotonicity is broken. Moreover, we observe that the optimal transmit probabilities are almost the same when $Q_{I I}$ is small. 


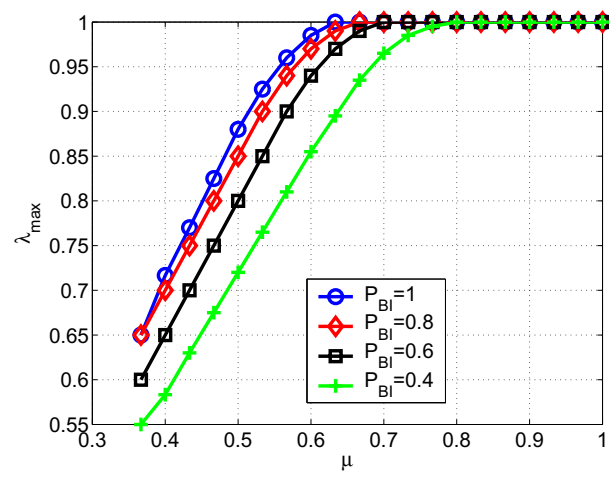

Fig. 3: Maximal arrival rate versus different service rates and different interruption durations.

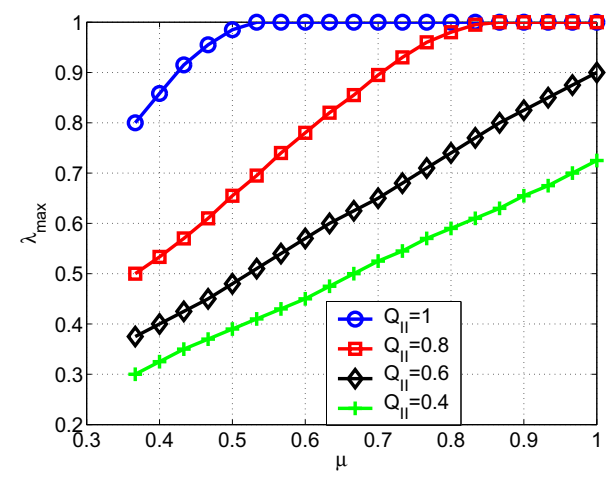

Fig. 4: Maximal arrival rate versus different service rates and different interruption arrival rates.

\section{CONCLUSIONS}

In this paper, we have analyzed the queuing system under Markovian interruptions, which is a good model for the data traffic in cognitive radio networks. We have discussed two special cases, namely two-server-single-queue and two-queuessingle-server. For the former case, we have obtained a semianalytic expression for the generating function of queue length. For the latter case, we have obtained the equations determined the average queue length (thus determining the average waiting time according to Little's Law). For the stability of the queuing system, we have used numerical simulations to obtain the maximum arrival rate that stabilizes the queue(s) with respect to different service rates and interruption parameters.

\section{REFERENCES}

[1] F. Borgonovo, M. Cesana and L. Fratta, "Throughput and delay bounds for cognitive transmissions," Advances in Ad hoc Networks, pp. 179190, Aug. 2008.

[2] B. T. Doshi, "Queuing systems with vacations - A survey," Queuing Systems, pp. 29-66, 1986.

[3] D. Fiems, T. Maertens and H. Bruneel, "Queuing systems with different types of server interruptions," European Journal of Operational Research, vol.188, pp. 838-845, Aug. 2008.

[4] D. P. Gaver Jr, "A waiting line with interrupted service, including priorities," Journal of the Royal Statistical Society, vol.24, pp. 73-90, 1962.

[5] E. Hossain, D. Niyato and Z. Han, Dynamic Spectrum Access in Cognitive Radio Networks. Cambridge University Press, UK, 2009

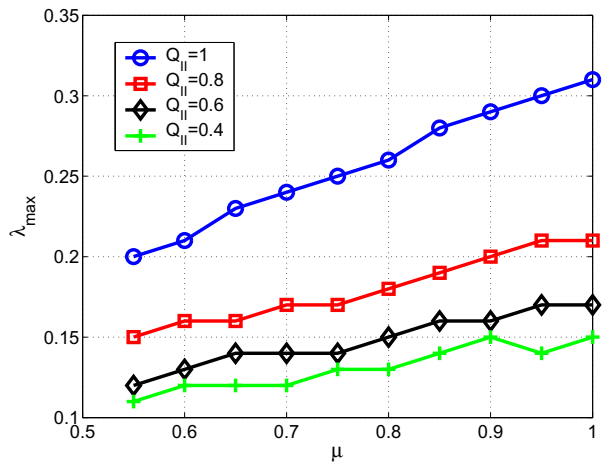

Fig. 5: Maximal arrival rate versus different service rates and different interruption arrival rates.

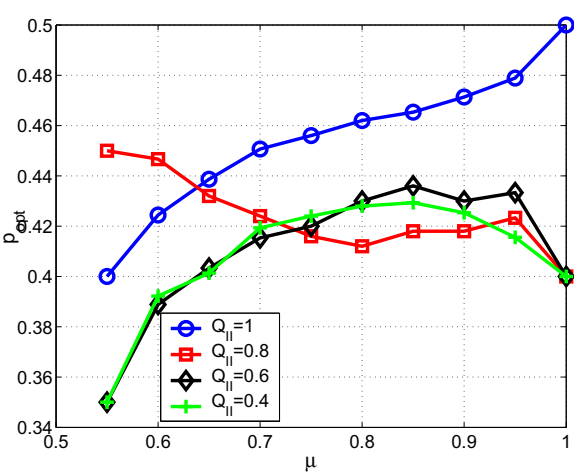

Fig. 6: Optimal transmit probability $p$ versus different service rates and different interruption arrival rates.

[6] J. Mitola, "Cognitive radio for flexible mobile multimedia communications," in Proc. IEEE Int. Workshop Mobile Multimedia Communications, pp. 3-10, 1999.

[7] A. Sahai, R. Tandra, M. Mishra and N. Hoven, "Fundamental design tradeoffs in cognitive radio systems," ACMoog TAPAS, Aug. 5, 2006.

[8] H. P. Shiang and M. Scharr, "Queuing-based dynamic channel selection for hetorogeneous multimedia applications over cognitive radio networks," IEEE Trans. Multimedia, vol.10, pp. 896-909, Aug. 2008.

[9] O. Simeone, Y. Bar-Ness and U. Spagnolini, "Stable throughput of cognitive radios with and without relaying capacbility," IEEE Trans. Commun., vol.55, pp. 2351-2360, Dec. 2007.

[10] H. Takagi and L. Kleinrock, "Mean packet queuing delay in a buffered two-user CSMA/CD system," IEEE Trans. Commun., vol.33, pp.11361139, 1985.

[11] S. Tang and B. L. Mark, "Analysis of opportunistic spectrum sharing with Markovian arrivals and phase-type service," IEEE Trans. Wireless Commun., to appear.

[12] N. Tian and Z. G. Zhang, Vacation Queuing Models: Theory and Applications, Springer, 2006.

[13] Q. Zhao and B. M. Sadler, "A survey of dynamic spectrum access," IEEE Signal Processing Magazine, vol. 24, pp. 79-89, May. 2007 